\title{
CHEMISCHE REVUE ÜBER die FEtT- UND Harz-INDUStrie
}

Technlsch-Wlssenschaftllches Zentralorgan

für dle Industrlen der Fette, Oele und Mineralöle, der Selfen-, Wachs-, Kerzen- und Lackfabrlkation, sowle der Harze.

Nachdrack der Originalartikel nur mit Genehmigung der Redaktion und mit rollstăndiger Quellenangabe gestattet.

XVII. Jahrgang.

H a m b a r g, Januar 1910.

Heft 1.

\section{Inhaltswerzeiehnis von Heft 1.}

Originalarbeiten : Uebor Seffonfabrikatioa, L. Ubbelohde. - Ueber die Volumen rerminderung des Paraffins belm Ersterren nnd Aaskristallisieren, Dr. Ed. Graefe. - Der Teergehalt in Palinnölen (Wagenachsen. Eisenbahnölen), Siegfried Lőwy. - Qnantitative Bestimmnng von Benzin in Terpentinöl, Dr. J. Marcusson. Zur Frage der Atichtigen Fettsouren belm Fettupaltangsprozess nach Twitchell, Oscar Steiner. - Zur Frago der Prüfung der Erdole anf Paraffn, K. W. Charitschkoft. - Berichte: Wissenschaftliches: Das Oel des Koloquintensamens, Dr. C. Grimaldi und L. Prussia. - Eln nener afrikanischer Kopal Erich Stock. - Synthese der hochsledenden Naphthene, N. Zielinsky. - Analyse: Bericht iber die Tistigkelt der Oelabtellung des rönlglichen Materialprifungsamtes in Gross-Lichterfelde-West im Betriehajahr 1308. - Aendernngen in der Zn. sammensetzung des Schwelneschmalzes, E. Durier. - Holde's Test zam Nachweis von Parafin in Schmalz uud anderen Fetten, H. Dunlop. - Fettbestimmang In Faeces, A. Chapus. - Der Flammpunkt als Hilfsaittel bei Wachsanalyen, Ed. Stoeber. - Chemische Untersuchung ron Asphalten, S. W. Parr. - Zur Bestimmung des Asphaltgehaltes In ZJlinderölen, Dr. R. Kiasling. - Apparat zun Filtrieren bel konstenter Temperator, Dr. Alfred Eisenstein und Friedrich Ziffer. - Deber die Wasser- und Schmandbestimmung in Erdölen, Dr. Rosenthal. - Technologies Asphalt and Bitumen und Jhro Verwendnng, A. Granger. - Salbenartige Schmierderivate an: Erdöl als Talgergatzstofio, Charitschkoff. - Frelo Fottgänren Im Talg. - Borsanres Mangan, Dipl.-Ing. Meister. - Erdöltorf. - Literatur. - Liste Deutscher Patente. - Deutsche Patentschriften. - Zollamtliches. - Verschiedenes. - Waren-Preis-Liste.

\section{Ueber Seifenfabrikation.}

Von L. Ubbelohde, Karlsruhe i. B. Technische Hochschnle.

Trotz der grossen Fortschritte und tiefgehenden Erkenntnis, welche die Chemie und Analyse der Fette uns gebracht hat, steht, wie wir wissen, eine der wesentlichsten Fettindustrien, die Seifenfabrikation, erst am Anfange einer Entwickelung nach der wissenschaftlichen Seite hin.

Zwar ist der Chemismus der Fettspaltung und Verseifung oft Gegenstand eingehender Arbeiten gewesen. Die Verseifung selbst, d. h. die Ueberführung der Fette in fettsaure Salze, ist aber nur ein Teil der seifensiederischen Tätigkeit. Ein anderer, ungleich vielseitigerer Teil ist die Arbeit, die auf die eigentliche Verseifung folgt; nämlich die Ueberführung der fettsauren Salze in die verkaufsfertigen Seifen, die typischen Produkte von bestimmtem Aussehen, bestimmter physikalischer Beschaffenheit, besonderem Wasser-, Salzgehalt usw. Diese Arbeit, die wir als das , Fertigmachen der Seifen. bezeichnen, ist bis vor kurzem kaum jemals Gegenstand systematischer wissenschaftlicher
Forschungen gewesen. Die Wissenschaft hatte die beim Fertigmachen der Seifen stattfindenden Vorgänge noch auf keine Formel zurückgeführt und sah auf diese Arbeit herab, wie auf eine handwerksmässige Fertigkeit, die in dieser oder jener Form zur Ausübung kommen könne.

Freilich, wenn wir die Literatur über Seifenfabrikation betrachten, so fallt darin eine ungewöhnlich grosse Summe von Einzelbeobachtungen über die Herstellung der einen oder anderen Seifenart aus verschiedenen Rohmaterialien auf, an die sich praktisch erprobte Vorschriften über ihre Herstellung knüpfen. Diese Beobachtungen stehen jedoch ziemlich zusammenhanglos da, so dass die gebräuchlichen Handbücher über Seifenfabrikation keinen sehr systematischen Aufbau, sondern mehr den Charakter eines Rezeptbuches zeigen. Unwillkürlich drängt sich beim Betrachten dieser Dinge die Frage auf, wie gewinnt man Seife aus einem noch unbekannten Rohmaterial, oder wie kann man willkürlich den Charakter der Seife verändern, 
Fragen, auf die ohne weiteres bis jetzt keine umfassende Antwort zu geben ist, da es an systematischen Kenntnissen hierüber vollkommen fehlt. An ihre Stelle tritt heutzutage noch ganz und gar die handwerksmässige Routine, bei der das Gefühlsmässige der in langjähriger praktischer Tätigkeit erworbenen ,Kunst* in den Vordergrund tritt.

Man hat von jeher bei der Seifenfabrikation das Fettsäuregemisch des Fettansatzes als Ganzes betrachtet. Dieses Gemisch ist aber von ungemein wechselnder Zusammensetzung, so dass es unmöglich wird, allgemeine Regeln für sein Verhalten aufzustellen, so lange man dies nicht in Beziehung zu seiner Zusammensetzung tut. Die Gesamtfettsäuren irgendeines Fettes oder auch des Fettansatzes für irgendeine Seife gleichen sich zwar im allgemeinen in ihrer qualitativen Zusammensetzung; sie bestehen nämlich in der Regel aus Palmitinsäure, Stearinsäure und Oelsäure, zu denen meist in geringerer Menge noch Linolsäure, Linolensäure und noch einige niedrigermolekulare gesättigte Säuren usw. hinzukommen. Die Gesamtfettsäuren unterscheiden sich jedoch sebr wesentlich in dem Mengenverhältnis der genannten Stoffe. Deshalb ist es erst dann möglich, einfache Gesichtspunkte in die verwickelten technischen Verhältnisse hineinzutragen, wenn man versucht, das Verhalten der Fettansätze zurückzuführen auf ihre Zusammensetzung, d. h. auf das Verhalten der einzelnen Fettsäuren für sich und in Gemischen miteinander.

Es ist das Verdienst François Merklen's ${ }^{1}$ ), darauf hingewiesen zu haben, dass zum Verständnis der Vorgänge, welche sich beim Fertigmachen der Seife abspielen, die Phasengesetze und die Kolloidchemie heranzuziehen sind. In seiner Arbeit, die in der vortrefflichen deutschen Uebersetzung von Dr. Franz Goldschmidt, Karlsruhe, vorliegt, zeigte er, dass die Seife aufzufassen ist als das $\mathrm{Gel}^{2}$ ) des Kolloids Seife, und dass die Mengen an Salz, Wasser, Alkali usw., die in der Seife enthalten sind, abhängig sind einerseits von der Konzentration der Unterlauge bezw. des Leimniederschlages an diesen Bestandteilen und von der Temperatur, andererseits aber auch in hervorragendem Masse von dem Charakter der Fettsäuren, welche die Seife enthält.

1) Die Kernseifen, ihre Zusammensetzung und Fabrikation vom Standpunkt der physikalischen Chemies. Uebersetzung ins Deutsche von Dr. Franz Goldschmidt, 1907. (Verlag von Wilhelon Knapp, Halle a. d. Saale).

3) Auf die Bedeutung dieses aus der Kolloidchemie stammenden Ausdrucks werden wir später noch zuríckkommen.
Durch Versuche in grossem Massstabe mit Fetten verschiedener Art konnte er nämlich nachweisen, dass die aus diesen stammenden Gesamtfettsäuren bei der Seifendarstellung wesentlich verschiedenen Charakter hinsichtlich der Empfindlichkeit gegen Elektrolyte beim Aussalzen und hinsichtlich der Adsorptionsfähigkeit für diese zeigten. Das heisst, Merklen gab eine wissenschaftliche Erklärung für denjenigen Vorgang, der sich beim ,Fertigmachen * der Seife abspielt, für den Bereich derjenigen Arbeit, welche die eigentliche handwerksmässige Kunst des Seifensieders umfasst.

So viel hierdurch in wissenschaftlicher Hinsicht auch erreicht ist, so müssen doch diese Untersuchungen für die praktische Seifenfabrikation so lange ohne tiefgreifenden Einfluss bleiben, als es nicht möglich ist, die vorliegenden Verhältnisse leichter zu übersehen und sie auf jeden einzelnen praktischen Fall ohne weiteres mehr oder weniger schablonenhaft zu übertragen. Bis dies erreicht ist, bleiben die bis jetzt vorliegenden Schwierigkeiten bestehen, denn die Ergebnisse der neuen wissenschaftlichen Erkenntnisse kann die Praxis in der bis jetzt vorliegenden Form nicht genügend anwenden.

Wie oben bereits erwähnt und wie aus der Merklen'schen Arbeit hervorgeht, ist das Verhalten der Fettansätze eine Funktion ihrer $\mathrm{Zu}$ sammensetzung. Auf diese muss man sie also zurückführen. Um dies aber zu können, sind umfangreiche systematische Arbeiten nötig, denn es ist zunächst festzustellen:

1. das Verhalten der reinen einzelnen Fettsäuren bei der Seifendarstellung; d. h. ihr Verhalten gegenüber allen in Frage kommenden Laugen, Salzlösungen und Gemischen derselben.

2. wie weit die einzelnen Fettsäuren ihre charakteristischen Eigentümlichkeiten auch in Mischung miteinander bewahren.

Nach Klarstellung dieser beiden Punkte würde man in der Lage sein, das Verhalten eines Fettsäuregemisches von bekannter $\mathrm{Zu}$ sammensetzung bei der Seifendarstellung vorauszusagen. Und da die physikalischen und chemischen Konstanten von Fettsäuregemischen ihre Zusammensetzung kennzeichnen, so wird man in der Lage sein, lediglich durch Prüfung der Fettsäuren im Laboratorium zu beurteilen, welcher Fabrikationsgang für sie der geeignetste ist, und welches Produkt sich dabei ergeben wird. Andererseits würde man, auf demsclben Wege rückwärts gehend, in der Lage sein, den geeigneten Fettansatz für eine Seife von bestimmter geforderter Art zusammenzustellen. 
Heutzutage ist für diese $Z$ wecke vielfach langes Herumprobieren nötig, um die geeigneten Bedingungen aufzufinden; eine Arbeit, die viel Geschicklichkeit und langjährige Erfahrung voraussetzt, vielfach aber nicht zum $Z$ iele führt, teils weil man die geeigneten Bedingungen nicht auffindet, teils aber auch, weil sie effektiv ausser dem Bereich des Möglichen liegen, dessen genaue Grenzen uns so lange unbekannt bleiben werden, als wir nicht systematische Untersuchungen der oben bezeichneten Art besitzen.

Diese sollen also zahlenmässige Grundlagen schaffen für allgemein gültige Gesetze, Grundlagen, wie sie für andere wissenschaftliche $Z$ weige zum Teil schon vorliegen, und ermöglichen, jeden einzelnen $F$ all vorauszubestimmen. Auf vielen Gebieten der physikalischen Chemie ist man noch damit beschäftigt, solche allgemeine zahlenmässige Beziehungen festzustellen, und eine ähnliche Arbeit stellt auch diejenige vor, welche von meinen Mitarbeitern im Chemisch-technischen Institut der Technischen
Hochschule in Karlstube seit 1908 in Angriff genommen ist. Sie wird zwar noch geraume Zeit zu ihrer völligen Durchführung in Anspruch nehmen, doch soll in der Folge damit begonnen werden, hierüber zu berichten, soweit ein gewisser Abschluss gewonnen ist.

Laboratoriumsmethode $z u r$

Bestimmung des Verhaltens der Fettsäuren bei der Seifendarstellung in Gemeinschaft mit Dipl.-Ing. The odor Richert.

Merklen hatte seine oben erwähnten Versuche in halbgrossem Massstabe durchgeführt durch jedesmalige Verseifung von $100 \mathrm{~kg}$ Oel. Dieses Verfahren liess sich für unsere Zwecke nicht verwenden; es musste vielmehr angestrebt werden, die Beobachtungen wegen Zeit- und Materialersparnis mit kleinen Mengen im Laboratorium durchzuführen, da in Anbetracht der notwendigen ungewöhnlich zahlreichen Versuche möglichste Einfach heit derselben angestrebt werden musste.

(Fortsetzung folgt.)

\section{Ueber die Volumenverminderung des Paraffins beim Erstarren und Auskristallisieren. \\ Von Dr. Ed. Graefe, Webau.}

Die meisten Stoffe, die vom geschmolzenen Zustand in den festen übergehen können, zeigen dabei eine beträchtliche Abnahme des Volumens, nur wenige, und darunter wohl der bekannteste das Wasser, zeigen das umgekehrte Verhalten.

Im allgemeinen muss man wohl bei technisch verwertbaren Stoffen diese Eigenschaft der Kontraktion als einen Nachteil bezeichnen, da sie besondere Massregeln erfordert, ihr zu begegnen, es sei nur an die Notwendigkeit des sog. verlorenen Kopfes beim Metallgiessen erinnert, nur in wenigen Fällen ist sie erwünscht. In der Kerzengiesserei ist sie jedenfalls äusserst unerwünscht, da sie nötigt, in die Giesspfannen der Kerzengiessmaschinen stets einen grossen Ueberschuss von flüssigem Kerzenmaterial zu giessen, aus dem dann das erstarrende Material in der Form soviel nachsaugt, um die infolge der Kontraktion entstehende Volumendifferenz auszugleichen. Man kann diesen zuzufügenden Ueberschuss, der die sog. "Decke» bildet, je nach der Grösse der Kerze auf etwa 30-80\% des nutzbaren Materials veranschlagen. Es lässt sich leicht nachweisen, dass der Extraaufwand den das Wiederaufschmelzen der Decken veranlasst, die natürlich wieder als Kerzenmaterial eingeschmolzen werden, ferner der grössere Dochtverlust, da man die Kerzen über die Decke heben muss, und die Materialverschlechterung durch das öftere Wiederaufschmelzen für eine Kerzenfabrik von etwa 100 Doppelzentner tägliche Leistung auf weit mehr als 10000 Mark im Jahr zu veranschlagen sind. Nun wird man ja an der Eigenschaft des Paraffins nichts ändern können und nur versuchen, ihrer nachteiligen Wirkung zu begegnen, aber es wäre doch immerhin möglich, unter den verschiedenen Paraffinen solche auszusuchen und zu bevorzugen, die diese Eigenschaft in geringerem Grade zeigen, falls überhaupt Abstufungen vorhanden sind.

In der Literatur findet man nur wenig über diesen Gegenstand. So erwähnt nur Scheithauer in seinem Buche über die Fabrikation der Mineralöle, dass die Ausdehnung beim Parafin beim Uebergang vom festen in den flüssigen Zustand etwa $1 / 7-1 / 9$ seines Volumens beträgt, doch sind diese Angaben fast ausschliesslich auf Braunkohlenteerparaffine bezogen und nicht für besondere Fälle zahlenmässig festgelegt. Petrolparaffine kamen zur Zeit, als die bei Scheithauer angeführten Daten gefunden wurden, noch wenig in Betracht, während sie jetzt aus den verschiedensten Rohölen gewonnen werden. Nachstehende Angaben bezwecken, die Lücke wenigstens teilweise auszufüllen. Es kamen zur Verwendung Petrolparaffine, Braunkohlenteerparaffine, Stearine und 